\title{
Shaping of Regional Differences in Oligodendrocyte Dynamics by Regional Heterogeneity of the Pericellular Microenvironment
}

\author{
Amin Sherafat ${ }^{1}$, Friederike Pfeiffer ${ }^{1,2}$ and Akiko Nishiyama ${ }^{1,3,4 *}$ \\ ${ }^{1}$ Department of Physiology and Neurobiology, University of Connecticut, Storrs, CT, United States, ${ }^{2}$ Department of \\ Neurophysiology, Institute of Physiology, Eberhard Karls University of Tübingen, Tübingen, Germany, ${ }^{3}$ Institute of Systems \\ Genomics, University of Connecticut, Storrs, CT, United States, ${ }^{4}$ The Institute of Brain and Cognitive Sciences, University of \\ Connecticut, Storrs, CT, United States
}

OPEN ACCESS

Edited by:

Peggy Assinck,

University of Edinburgh,

United Kingdom

Reviewed by:

Mark R. Kotter,

University of Cambridge,

United Kingdom

Davide Lecca,

University of Milan, Italy

*Correspondence:

Akiko Nishiyama

akiko.nishiyama@uconn.edu

Specialty section: This article was submitted to

Non-Neuronal Cells,

a section of the journal

Frontiers in Cellular Neuroscience

Received: 06 June 2021 Accepted: 31 August 2021 Published: 08 October 2021

Citation:

Sherafat $A$, Pfeiffer $F$ and Nishiyama A (2021) Shaping of Regional Differences in Oligodendrocyte Dynamics by Regional Heterogeneity of the Pericellular Microenvironment. Front. Cell. Neurosci. 15:721376. doi: 10.3389/fncel.2021.721376
Oligodendrocyte precursor cells (OPCs) are glial cells that differentiate into mature oligodendrocytes (OLs) to generate new myelin sheaths. While OPCs are distributed uniformly throughout the gray and white matter in the developing and adult brain, those in white matter proliferate and differentiate into oligodendrocytes at a greater rate than those in gray matter. There is currently lack of evidence to suggest that OPCs comprise genetically and transcriptionally distinct subtypes. Rather, the emerging view is that they exist in different cell and functional states, depending on their location and age. Contrary to the normal brain, demyelinated lesions in the gray matter of multiple sclerosis brains contain more OPCs and OLs and are remyelinated more robustly than those in white matter. The differences in the dynamic behavior of OL lineage cells are likely to be influenced by their microenvironment. There are regional differences in astrocytes, microglia, the vasculature, and the composition of the extracellular matrix (ECM). We will discuss how the regional differences in these elements surrounding OPCs might shape their phenotypic variability in normal and demyelinated states.

Keywords: myelin, NG2, PDGF, blood vessels, astrocyte, microglia, neuropilin

\section{INTRODUCTION}

Oligodendrocyte precursor cells (OPCs), also known as NG2 glia or polydendrocytes, are uniformly distributed throughout the gray and white matter of the adult central nervous system (CNS). They are lineage committed precursor cells whose primary known function is to differentiate into mature oligodendrocytes (OLs) that myelinate axons and enable fast saltatory conduction of action potentials. OPCs express the cell surface proteins NG2 and platelet-derived growth factor receptor alpha (PDGFR $\alpha$ ), which are downregulated during their terminal differentiation into postmitotic oligodendrocytes. The term NG2 cells or NG2 glia is used synonymously with OPCs, regardless of whether the cell differentiates into an OL or continues to exist as an OPC for an extended period of time, as currently evidence is lacking that OPCs comprise cells with dichotomous OL differentiation dynamics. 
OPCs arise from discrete germinal zones in the embryo and continue to be generated from the subventricular zone in the mature CNS. Despite the reports that nearly all OPCs are in active cell cycle state in the adult brain (Rivers et al., 2008; Psachoulia et al., 2009; Kang et al., 2010), OPCs are heterogeneous in their proliferative rate. In the normal rodent CNS, OPCs proliferate and differentiate into OLs at a higher rate in the white matter than in the gray matter (Hill and Nishiyama, 2014; Boshans et al., 2020; Nishiyama et al., 2021; Figure 1, left). Myelination continues throughout life beyond developmental myelin production and appears to be finely tuned to the local neural network (Monje and Karadottir, 2020; Nishiyama et al., 2021; Pease-Raissi and Chan, 2021).

Damage to myelin occurs in demyelinating diseases such as multiple sclerosis (MS; Lassmann, 2019). Poor remyelination of chronic MS lesions could result from failure of OLs to myelinate axons (Chang et al., 2002) or defects in OPCs (Jäkel et al., 2019; Yeung et al., 2019). Based on the OL dynamics in the normal brain, one would predict that MS lesions in the white matter would be more efficiently remyelinated. Contrary to this prediction, OL dynamics during remyelination in MS is more robust in gray matter lesions (Peterson et al., 2001), despite a higher density of OPCs and OLs in normal appearing white matter than in normal appearing gray matter of MS brains (Strijbis et al., 2017). In this mini review, we will discuss how the regional heterogeneity of the microenvironment surrounding OL lineage cells might contribute to their region-specific behavior in normal and pathological states.

\section{REGIONAL HETEROGENEITY OF OL LINEAGE CELLS}

It has been debated as to whether OPCs represent a heterogeneous cell population (Dimou and Simons, 2017; Foerster et al., 2019; Boshans et al., 2020). Unlike neurons which can be classified into functionally and transcriptionally distinct cell types, OPCs exhibit a spectrum of phenotypes that varies with the age and anatomical location. Below are examples of the phenotypic variability among OPCs and an emerging hypothesis that these differences represent different cell and functional states rather than transcriptionally distinct subtypes.

\section{OPC Distribution Does Not Always Parallel OL Distribution}

While OPCs are distributed throughout the CNS, their density is higher in white matter than in gray matter (Dawson et al., 2000, 2003; Terai et al., 2003). One determinant of OPC density could be the necessity to generate OLs. Consistently, OPC density is low in CNS regions where unmyelinated axons predominate such as the molecular layer of the cerebellar cortex (Givogri et al., 2002; Sun et al., 2018). However, in other regions the distribution of OPCs does not match that of OLs. For example, in the neocortex OPC are distributed uniformly throughout all cortical layers, whereas myelinating OLs are more abundant in deeper cortical layers (Tomassy et al., 2014). Curiously, OPCs exist in relatively high density in some circumventricular organs (Terai et al., 2003; Zilkha-Falb et al., 2020), where capillaries are fenestrated and their density is high (Gross et al., 1986), suggesting a possible non-progenitor role for OPCs. Indeed, OPCs are involved in feeding and body weight control by modulating leptin-sensing of leptin receptorexpressing neurons in the arcuate nucleus that extend their dendrites into the adjacent median eminence (Djogo et al., 2016).

\section{Higher Rate of OPC Proliferation and OL Differentiation in White Matter}

OPCs in white matter proliferate and generate OLs at a greater rate than those in the gray matter (Figure 1, left; Dawson et al., 2003; Dimou et al., 2008; Psachoulia et al., 2009; Kang et al., 2010; Zhu et al., 2011; Young et al., 2013). In slice culture, OPCs in the white matter of the developing corpus callosum and cerebellum proliferate more robustly in response to exogenous PDGF AA than those in the neocortex and cerebellar cortex, despite similar levels of PDGFR $\alpha$ (Hill et al., 2013). When OPCs are isolated from adult gray and white matter and transplanted into gray and white matter of recipient adult mice (Vigano et al., 2013), those from the white matter but not gray matter differentiate robustly into OLs in the host gray matter, whereas those from both gray and white matter differentiate into OLs in the host white matter, suggesting both intrinsic and environmental influences. In slice culture, homoand heterotopic transplantation of $300-\mu \mathrm{m}^{3}$ explants or isolated explant cultures indicated that the proliferative response of OPCs to PDGF AA is determined by signals within the 300$\mu \mathrm{m}^{3}$ microenvironment around the OPCs (Hill et al., 2013). A recent follow-up study demonstrated that neuropilin-1 (Nrp1), a co-receptor for PDGFR $\alpha$, is expressed by microglia adjacent to OPCs in white but not gray matter and trans-activates PDGFR $\alpha$ on OPCs (Sherafat et al., 2021), providing an example of a regional microglial difference affecting OPC dynamics (see

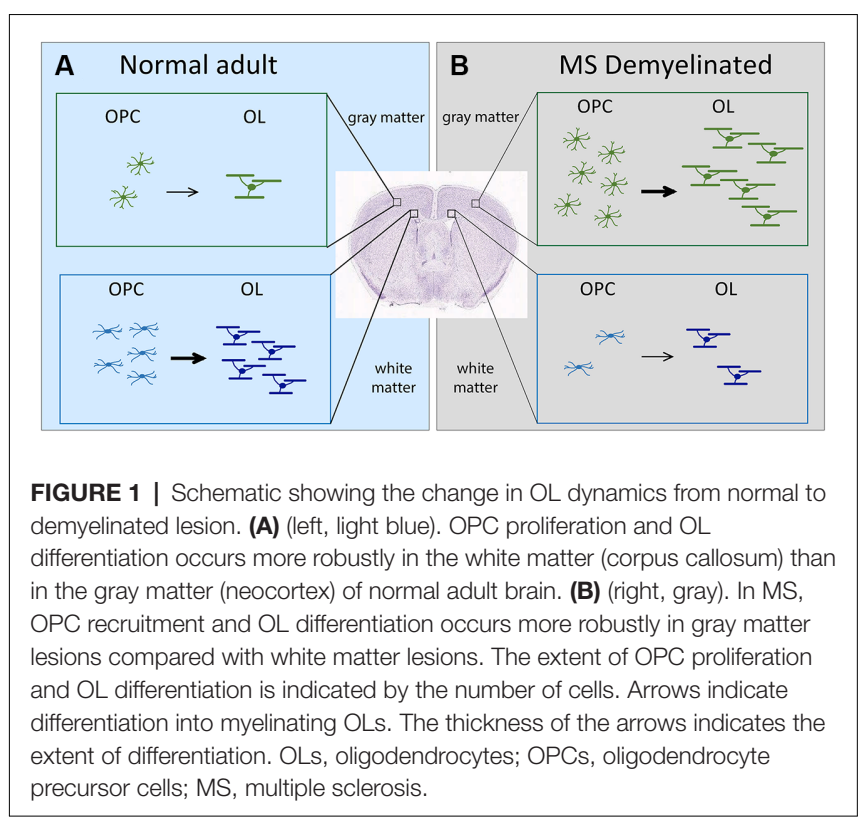


section "Regional Heterogeneity of Microglia Affects OL Lineage Cells" below).

\section{OPCs Are Transcriptionally Homogeneous}

Single-cell RNA-sequencing (scRNA-seq) has revealed multiple subtypes of OLs, but OPCs are more transcriptionally homogeneous and fail to cluster into transcriptionally distinct subtypes (Zeisel et al., 2015; Marques et al., 2016; Tasic et al., 2016). In scRNA-seq of OPCs from different ages, the OPC cluster that expresses genes involved in mitosis segregates from the other two clusters found, which express similar transcripts but appear in postnatal day 7 (P7) and juvenile/adult CNS, respectively (Marques et al., 2018). These clusters are not sufficiently distinct to allow classification of OPCs into distinct subpopulations but may represent different cell states described in the next section.

\section{Different Cellular and Functional States of OPCs}

OPCs in gray and white matter exhibit different electrophysiological properties shaped by the density of voltagedependent $\mathrm{Na}^{+}$and $\mathrm{K}^{+}$channels $\left(\mathrm{Na}_{\mathrm{v}}\right.$ and $\mathrm{K}_{\mathrm{v}}$, respectively; Chittajallu et al., 2004; Clarke et al., 2012; Larson et al., 2016). A recent comprehensive analysis of $\mathrm{Na}_{\mathrm{v}}, \mathrm{K}_{\mathrm{v}}$, and glutamate receptor expression and membrane properties of individual OPCs in the cortex and corpus callosum at different ages revealed unique patterns as a function of age and location (Spitzer et al., 2019). When these data are projected against other cellular properties and transcriptional profiles, some correlations emerge. For example, proliferative OPCs have a higher $\mathrm{Na}_{\mathrm{v}}$ density, and NMDAR expression coincides temporally with OL differentiation. From these analyses, Karadottir and colleagues propose that OPCs can exist in several different states that include: (1) naive state with no voltage-dependent ion channel expression; (2) proliferative state with high $\mathrm{Na}_{\mathrm{v}}$ density and a transcriptomic profile of mitotic cells; (3) state primed for OL differentiation with high NMDAR density and higher transcripts for $\mathrm{OL}$ and myelin genes; and (4) quiescent state with reduced $\mathrm{Na}_{\mathrm{v}}$ and NMDAR density (Kamen et al., 2021). These states are likely to be affected by their local environment. It is currently not known what specific signals drive them to transition from one state to another, the level of plasticity and convertibility among different states, and how the different OPCs states in turn influence the local neural network.

\section{HETEROGENEITY OF THE PERICELLULAR ENVIRONMENT AROUND OL LINEAGE CELLS}

Many excellent reviews have been published on how neuronal activity influences OL lineage cell behavior. Here, we will discuss how non-neuronal elements might affect the behavior of OL lineage cells. Recent studies have revealed regional heterogeneity of astrocytes and microglia. Furthermore, the vascular supply and vascular mural cell function are also likely to contribute to the different behavior of OL lineage cells in the cortex and corpus callosum.

\section{Regional Heterogeneity of Astrocytes Affects OL Lineage Cells}

Astrocytes are involved in a variety of functions from homeostasis of extracellular $\mathrm{K}^{+}$and glutamate to the regulation of synapses (Khakh and Sofroniew, 2015), and they influence $\mathrm{OL}$ development and function in a variety of ways (Barnett and Linington, 2013; Lundgaard et al., 2014; Tognatta et al., 2020). In response to a variety of insults, astrocytes become reactive and upregulate different sets of transcripts depending on the nature of the insults (Escartin et al., 2021). Transcriptional profiling showed that cortical astrocytes are enriched in genes required for cholesterol metabolism, and gray matter astrocytes support in vitro myelination more than 2 -fold compared with white matter astrocytes (Werkman et al., 2021). Cholesterol is critical for myelination (Saher et al., 2005). Since cholesterol does not cross the blood-brain barrier, cholesterol must be made available to OLs autonomously by biosynthesis in OLs and by horizontal transfer from astrocytes (Camargo et al., 2017). In mice with experimental autoimmune encephalitis (EAE), astrocytes express lower levels of transcripts necessary for cholesterol biosynthesis (Itoh et al., 2018), and astrocytes from MS brains have lower expression of cholesterol biosynthesis genes.

By contrast, astrocytes from the white matter of normal young adult rats express extracellular matrix (ECM) genes more abundantly than gray matter astrocytes (Werkman et al., 2020; Figure 2). ECM genes enriched in white matter astrocytes include ECM genes that are upregulated in reactive astrocytes, such as CD44 and collagen genes though they are expressed at a lower level in normal white matter (Zamanian et al., 2012). CD44 is a receptor for the large extracellular proteoglycan hyaluronan which inhibits OL maturation (Buser et al., 2012). Thus, astrocytes from adult white matter appear to be less supportive of myelination compared to those from gray matter. By contrast, those in the gray matter appear to metabolically supportive of OLs and myelin production.

\section{Regional Heterogeneity of Microglia Affects OL Lineage Cells}

Microglia and border-associated macrophages make up the tissue macrophages of the CNS (Prinz et al., 2021). Microglia exhibit regional heterogeneity in their morphology and density (Lawson et al., 1990). They are highly plastic cells that switch from a resting homeostatic phenotype to an activated phagocytic state triggered by a variety of insults including MS lesions (Lassmann et al., 2001). In experimentally induced demyelination, activated microglia initially contribute to demyelination (Marzan et al., 2021) but are subsequently replaced by repair-promoting microglia (Lloyd et al., 2019). Microglia not only sense pathology but also play essential roles in normal development and homeostatic processes, such as neurogenesis, synaptic pruning, and regulation of neuronal activity (Butovsky and Weiner, 2018; Thion et al., 2018; Badimon et al., 2020).

Recent studies suggest that there are region-specific interactions between microglia and OL lineage cells. The 


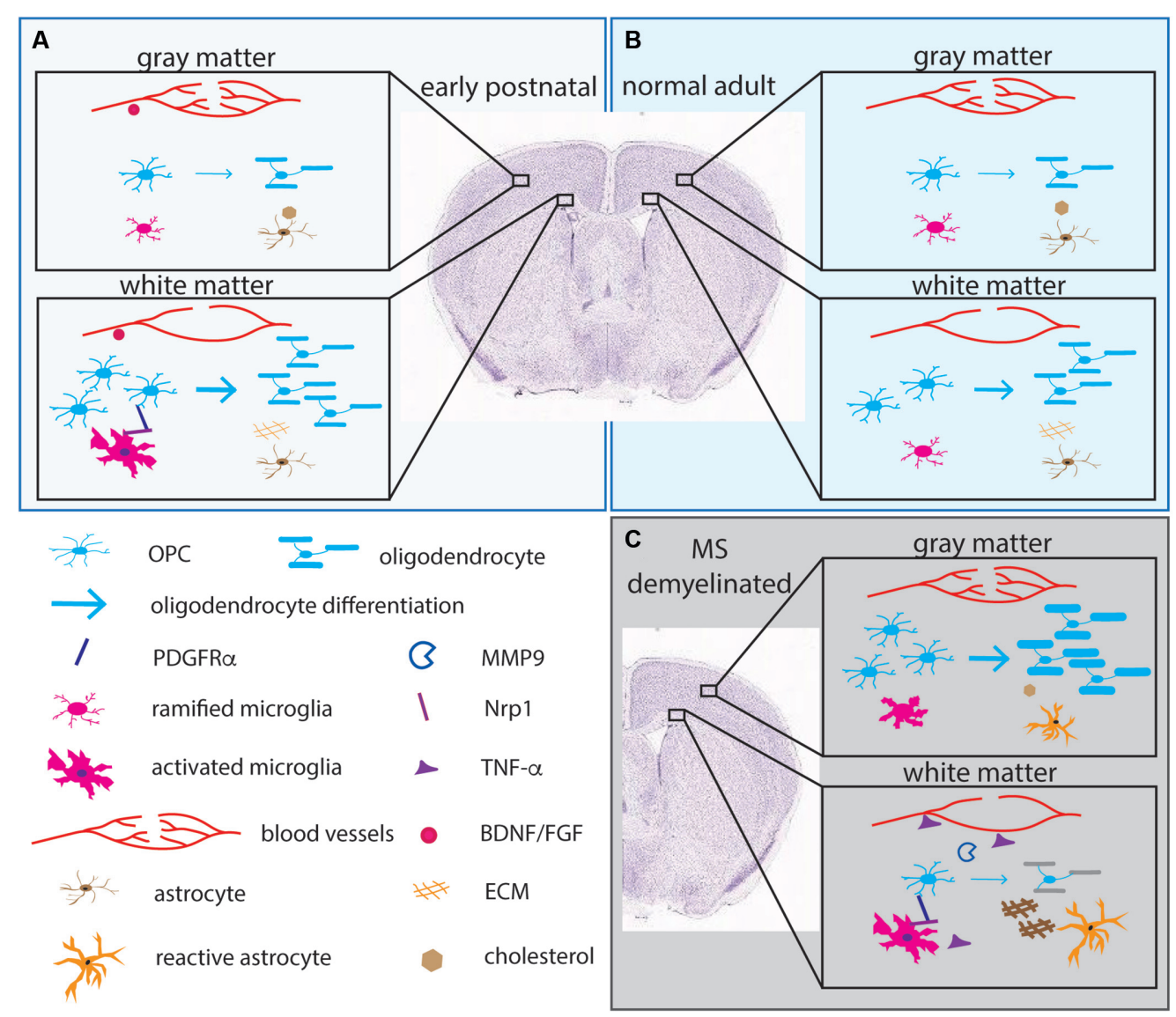

FIGURE 2 | The pericellular microenvironment affecting OPC dynamics under different conditions. (A) (upper left box, pale blue). Early postnatal brain (B) (upper right box, light blue). Normal adult brain (C) (lower right box, gray). Demyelinated lesions in MS brain or experimentally demyelinated lesions. OL lineage cells and their protein products (PDGFR $\alpha$, MMP9) are depicted in shades of blue. Microglia and their products (Nrp1, TNF- $\alpha$ ) are indicated in shades of pink. Astrocytes and their products (cholesterol, ECM) are indicated in orange/brown. Blood vessels and their products (BDNF, FGF) are in red. Arrows indicate differentiation into myelinating OLs. The thickness of the arrows indicates the extent of differentiation. The thick myelin in the gray matter in (C) represents successful remyelination. The gray myelin in the white matter in $\mathbf{( C )}$ represents failure to remyelinate in white matter. The depiction is meant to illustrate examples of the differences and is not meant to be a comprehensive illustration. ECM, extracellular matrix; FGF, fibroblast growth factor; BDNF, brain-derived neurotrophic factor; PDGFR $\alpha$, platelet-derived growth factor receptor alpha; MMP9, matrix metalloproteinase-9; Nrp1, neuropilin-1; TNF- $\alpha$, tumor necrosis factor alpha.

early postnatal (P1-P8) corpus callosum contains amoeboid microglia, also referred to as the "fountain of microglia". These are morphologically distinct from the ramified microglia in the cortex and have the transcriptional signature of activated phagocytic cells (Hagemeyer et al., 2017; Figure 2). They are likely to correspond to the phagocytic microglia seen by electron microscopy that were seen to have ingested "spongioblasts", which are likely to have been OPCs (Ling, 1976). Depletion of microglia with an antagonist to colony stimulating factor 1 receptor (CSF1R) during the first postnatal week reduces the number of OPCs and OLs (Hagemeyer et al., 2017). Another study showed that a similar population of activated microglia in the early postnatal corpus callosum that express CD11c secrete insulin-like growth factor 1 (IGF1) and play a critical role in developmental myelination (Wlodarczyk et al., 2017). Subsequently published two scRNA-seq studies revealed a microglial subtype that exists in the postnatal white matter that is transcriptionally distinct from developing cortical microglia or adult resting/homeostatic microglia (Hammond et al., 2019; Li et al., 2019). These cells have been termed axon-tract-associated microglia (ATM; Hammond et al., 2019) or proliferativeregion-associated microglia (PAM; Li et al., 2019). They express CD68 and other newly identified signature genes such as Spp1 and Clec7A.

Independently, we have shown that activated microglia in the postnatal white matter (ATM/PAM) but not ramified microglia in the cortex express neuropilin-1 (Nrp1), a co-receptor for vascular endothelial cell growth factor (VEGF; Pellet-Many et al., 2008) as well as PDGFR $\alpha$ (Ball et al., 2010; Sherafat et al., 2021). After a demyelinating injury, Nrp1 is strongly upregulated on activated microglia, and microglial Nrp1 deletion severely compromises OPC recruitment and subsequent remyelination (Sherafat et al., 2021). Furthermore, exogenous Nrp1 promotes PDGF AA-mediated OPC proliferation, most potently under 
conditions of limited amounts of PDGF AA, and exogenous Nrp1 increases tyrosine phosphorylation of PDGFR $\alpha$ on OPCs (Sherafat et al., 2021), suggesting that Nrp1 on ATM/PAM activates PDGFR $\alpha$ on adjacent OPCs in trans (Figure 2). While the mechanism that causes the ATM/PAM to adopt an activated phenotype in the early postnatal corpus callosum remains unknown, apoptosis of early-born ventrally derived OPCs (Orduz et al., 2019) or a sudden local drop in OPC density due to their rapid OL differentiation could trigger their appearance. Future studies could be directed toward elucidating the molecular signaling mechanisms that lead to the emergence of ATM/PAMs in the developing white matter in the context of a homeostatic process for maintaining the correct level of myelin and myelinating cells in the CNS. In demyelinated lesions, the tissue-damaging pro-inflammatory effects of activated microglia, including secretion of inflammatory cytokines such as tumor necrosis factor alpha (TNF- $\alpha$ ) are offset by the repair-promoting functions of microglia in a finely coordinated fashion (Lloyd et al., 2019; Figure 2).

\section{Regional Heterogeneity of Brain Microvessels and the Oligovascular Niche}

Evidence is accumulating for a functional interplay between OL lineage cells and the vasculature (Figure 2). In the developing CNS, OPCs migrate along blood vessels (Tsai et al., 2016), and factors from endothelial cells such as fibroblast growth factor 2 (FGF2) and brain-derived neurotrophic factor (BDNF) promote OPC survival and proliferation (Arai and Lo, 2009). Reciprocally, under pathological conditions such as inflammation, OPCs secrete matrix metalloproteinase-9 (MMP9), which increases blood-brain barrier permeability and in turn trigger further tissue damage such as demyelination (Seo et al., 2013). This has led to the concept of the "oligo-vascular niche", which extends the original concept of the "neurovascular niche" to include OL lineage cells. Recent findings suggest that there is reciprocal signaling between OPCs and vascular mural cells (Miyamoto et al., 2014; Kishida et al., 2019), and that neuronal activity affects myelination through altered cerebral blood flow (Swire et al., 2019).

In rat, the capillary density of the cortex is $3-5$ times greater than that of the white matter and correlates with glucose utilization (Borowsky and Collins, 1989; Figure 2). As pial capillaries perforate the cortex during embryonic development, they bring with them meningeal mesenchymal tissue which remains in the adult as the Virchow-Robin complex (MarinPadilla, 2012). These regional differences in the capillary network could contribute to the regional differences in the functions of OL lineage cells. We recently reported that $94 \%$ of OPC processes have close contact with blood vessels in the neocortex of adult mice, and conversely $92 \%$ of the vascular segments are contacted by OPC processes (Pfeiffer et al., 2021), suggesting a significant functional interaction between OPCs and vascular mural cells. It remains to be determined whether there are regional differences in the extent of physical relation between OPCs and the vasculature and the functional significance of the OPC-vascular contacts.

\section{REGIONAL HETEROGENEITY IN MS LESIONS}

Since the original description of patients with MS by Jean-Martin Charcot ${ }^{1}$ in 1868 and an earlier drawing by Sir Robert Carswell in $1838^{2}$, MS has been known as a disease that affects the white matter, causing demyelination and subsequent scarring and atrophy. More recent studies indicate that MS also affects the gray matter (Brownell and Hughes, 1962). Myelin basic protein immunohistochemistry revealed frequent cortical demyelinated lesions in autopsied brains from active (Peterson et al., 2001) and chronic (Albert et al., 2007) MS patients. Cortical lesions are more commonly seen in a subpial location and contain fewer inflammatory cells. Furthermore, the extent of cortical demyelination is significantly greater in patients with secondary progressive disease of long duration and in primary progressive MS, and pathological hallmarks for neuronal degeneration and apoptosis are present in cortical lesions (Peterson et al., 2001). The subpial cortical demyelination occurs specifically in MS and not in other conditions similar inflammatory mediators and oxidative tissue damage are involved (Junker et al., 2020).

Despite the widespread nature of cortical demyelination, remyelination is significantly greater in cortical lesions than in white matter lesions, particularly in chronic lesions (Albert et al., 2007). This is accompanied by a greater abundance of OPCs and OLs in cortical lesions compared to white matter lesions (Albert et al., 2007; Chang et al., 2012; Figure 1). Curiously, the converse is true for normal-appearing tissues in MS brains, where OPCs and OLs are found in greater numbers in normal appearing white matter than in normal appearing gray matter (Strijbis et al., 2017), similar to their distribution in normal rodent brains.

What makes OPCs in the gray matter more competent to respond to demyelinated lesions and promote repair? Some observations suggest inherent differences between OPCs in the cortex and white matter in their ability to respond to inflammatory and recruitment signals. For example, OPCs isolated from rat gray matter are more immature and are less responsive to the inhibitory effects of inflammatory cytokines such as interferon gamma (IFN- $\gamma$ ) and TNF- $\alpha$ on OL differentiation (Lentferink et al., 2018). It is also interesting to note that OPCs that develop later from dorsal germinal zones are recruited more robustly to demyelinated lesions than ventrally derived OPCs (Crawford et al., 2016). A recent scRNA-seq study on OL lineage cells from the white matter of postmortem brain from MS patients revealed a significant reduction in the number of cells with the gene expression profiles of OPCs and intermediate cells that are between OPCs and newly formed premyelinating OLs (Jäkel et al., 2019). Perhaps, gray matter OPCs that have not gone through as many rounds of replication as white matter OPCs suffer less from replicative senescence. A transcriptomic comparison between OL lineage

\footnotetext{
${ }^{1}$ Charcot JM. Leçons de 1868; Manuscrits des leçns de JM Charcot.: Fonds numérisé Charcot. Bibliothèque de l'Université Pierre \& Marie Curie (http://jubilotheque.upmc.fr/subset.html?name=collections\&id=charcot).

${ }^{2}$ Pathological Anatomy Illustrations of the Elementary Forms of Disease, London: 1838. https://www.gla.ac.uk/myglasgow/library/files/special/exhibns/month/ oct2003.html.
} 
cells in gray and white matter MS lesions may provide some answers to this.

On the other hand, there is quite strong evidence that suggests that environmental factors play a major role in shaping OL lineage cell dynamics that differ between gray and white matter MS lesions. White matter MS lesions contain more extensive reactive astrogliosis and more abundant inhibitors of myelination such CD44 and hyaluronan, many of which are produced by astrocytes (Chang et al., 2012). In addition, inflammatory cells including leukocytes and microglia are more prevalent in white matter lesions and provide an importance source of ECM proteins that hinder myelin repair (Ghorbani and Yong, 2021). After acute demyelination in the white matter, the ability of activated microglia and macrophages to successfully undergo efflux of ingested cholesterol influences the efficiency of subsequent remyelination, and this declines with age (Cantuti-Castelvetri et al., 2018). Furthermore, sterol synthesis in phagocytes is necessary to activate LXR signaling to trigger cholesterol efflux and promote resolution of inflammation and subsequent myelin repair (Berghoff et al., 2021).

Intriguingly, the distribution of cortical lesions appears to coincide with the distribution of the vascular supply (Kidd et al., 1999). The meninges have recently been shown to contain a supply of glial progenitor cells that migrate into the CNS parenchyma and contribute to oligodendrogliogenesis (Dang et al., 2019). These progenitor cells from the meninges could be a more efficient source of remyelinating OLs for subpial cortical MS lesions than the neural progenitor cells in the subventricular zone. Future studies may be directed toward understanding how the meningeal inflammatory infiltrates in cortical MS lesions affect the ability of meningeal progenitor cells to migrate into the cortex and contribute to myelin repair. The greater abundance of

\section{REFERENCES}

Albert, M., Antel, J., Bruck, W., and Stadelmann, C. (2007). Extensive cortical remyelination in patients with chronic multiple sclerosis. Brain Pathol. 17, 129-138. doi: 10.1111/j.1750-3639.2006.00043.x

Arai, K., and Lo, E. H. (2009). An oligovascular niche: cerebral endothelial cells promote the survival and proliferation of oligodendrocyte precursor cells. J. Neurosci. 29, 4351-4355. doi: 10.1523/JNEUROSCI.0035-09.2009

Badimon, A., Strasburger, H. J., Ayata, P., Chen, X., Nair, A., Ikegami, A., et al. (2020). Negative feedback control of neuronal activity by microglia. Nature 586, 417-423. doi: 10.1038/s41586-020-2777-8

Ball, S. G., Bayley, C., Shuttleworth, C. A., and Kielty, C. M. (2010). Neuropilin1 regulates platelet-derived growth factor receptor signalling in mesenchymal stem cells. Biochem. J. 427, 29-40. doi: 10.1042/BJ20091512

Barnett, S. C., and Linington, C. (2013). Myelination: do astrocytes play a role?. Neuroscientist 19, 442-450. doi: 10.1177/1073858412465655

Berghoff, S. A., Spieth, L., Sun, T., Hosang, L., Schlaphoff, L., Depp, C., et al. (2021). Microglia facilitate repair of demyelinated lesions via post-squalene sterol synthesis. Nat. Neurosci. 24, 47-60. doi: 10.1038/s41593-020 $-00757-6$

Borowsky, I. W., and Collins, R. C. (1989). Metabolic anatomy of brain: a comparison of regional capillary density, glucose metabolism and enzyme activities. J. Comp. Neurol. 288, 401-413. doi: 10.1002/cne.902880304

Boshans, L. L., Sherafat, A., and Nishiyama, A. (2020). The effects of developmental and current niches on oligodendrocyte precursor dynamics and fate. Neurosci. Lett. 715:134593. doi: 10.1016/j.neulet.2019.134593 blood vessels in the cortex and their associated Virchow-Robin space could play an important pathophysiological role in the dynamics of OL lineage cells in the demyelinated cortex.

\section{CONCLUDING REMARKS}

Despite the phenotypic variation of OPCs in different neuroanatomical regions, there has so far been no definitive evidence that OPCs can be segregated into distinct subtypes based on permanent changes in gene expression. Rather, OPCs weave in and out of different cell and functional states depending on their physiological context and microenvironment. The specific signals that cause OPCs to alter their functional states have yet to be elucidated. All the elements surrounding OPCs, including neurons, non-neuronal cells, vascular cells, and the ECM exert specific effects on OPC dynamics, and the nature of these signals can change dramatically under certain pathological conditions.

\section{AUTHOR CONTRIBUTIONS}

AS wrote a draft of the manuscript. FP wrote a draft of the manuscript on the vasculature ("Regional Heterogeneity in MS Lesions" section). AN edited the manuscript. All authors contributed to the article and approved the submitted version.

\section{FUNDING}

This work was funded by NIH R01 NS073425 and NS116182 to $\mathrm{AN}$ and the European Union's Framework Program for Research and Innovation Horizon 2020 (2014-2020) under the Marie Sklodowska-Curie Grant Agreement No. 845336 to FP.

Brownell, B., and Hughes, J. T. (1962). The distribution of plaques in the cerebrum in multiple sclerosis. J. Neurol. Neurosurg. Psychiatry 25, 315-320. doi: 10.1136/jnnp.25.4.315

Buser, J. R., Maire, J., Riddle, A., Gong, X., Nguyen, T., Nelson, K., et al. (2012). Arrested preoligodendrocyte maturation contributes to myelination failure in premature infants. Ann. Neurol. 71, 93-109. doi: 10.1002/ana.22627

Butovsky, O., and Weiner, H. L. (2018). Microglial signatures and their role in health and disease. Nat. Rev. Neurosci. 19, 622-635. doi: 10.1038/s41583-0180057-5

Camargo, N., Goudriaan, A., van Deijk, A. F., Otte, W. M., Brouwers, J. F., Lodder, H., et al. (2017). Oligodendroglial myelination requires astrocytederived lipids. PLoS Biol. 15:e1002605. doi: 10.1371/journal.pbio. 1002605

Cantuti-Castelvetri, L., Fitzner, D., Bosch-Queralt, M., Weil, M. T., Su, M., Sen, P., et al. (2018). Defective cholesterol clearance limits remyelination in the aged central nervous system. Science 359, 684-688. doi: 10.1126/science. aan 4183

Chang, A., Staugaitis, S. M., Dutta, R., Batt, C. E., Easley, K. E., Chomyk, A. M., et al. (2012). Cortical remyelination: a new target for repair therapies in multiple sclerosis. Ann. Neurol. 72, 918-926. doi: 10.1002/ana.23693

Chang, A., Tourtellotte, W. W., Rudick, R., and Trapp, B. D. (2002). Premyelinating oligodendrocytes in chronic lesions of multiple sclerosis. $N$. Engl. J. Med. 346, 165-173. doi: 10.1056/NEJMoa010994

Chittajallu, R., Aguirre, A., and Gallo, V. (2004). NG2-positive cells in the mouse white and grey matter display distinct physiological properties. J. Physiol. 561, 109-122. doi: 10.1113/jphysiol.2004.074252 
Clarke, L. E., Young, K. M., Hamilton, N. B., Li, H., Richardson, W. D., and Attwell, D. (2012). Properties and fate of oligodendrocyte progenitor cells in the corpus callosum, motor cortex and piriform cortex of the mouse. J. Neurosci. 32, 8173-8185. doi: 10.1523/JNEUROSCI.0928-12.2012

Crawford, A. H., Tripathi, R. B., Richardson, W. D., and Franklin, R. J. M. (2016). Developmental origin of oligodendrocyte lineage cells determines response to demyelination and susceptibility to age-associated functional decline. Cell Rep. 15, 761-773. doi: 10.1016/j.celrep.2016.03.069

Dang, T. C., Ishii, Y., Nguyen, V., Yamamoto, S., Hamashima, T., Okuno, N., et al. (2019). Powerful homeostatic control of oligodendroglial lineage by PDGFRalpha in adult brain. Cell Rep. 27, 1073-1089. doi: 10.1016/j.celrep.2019. 03.084

Dawson, M. R., Levine, J. M., and Reynolds, R. (2000). NG2-expressing cells in the central nervous system: are they oligodendroglial progenitors? J. Neurosci. Res. 61, 471-479. doi: 10.1002/1097-4547(20000901)61:5<471::AID-JNR1>3. $0 . \mathrm{CO} ; 2-\mathrm{N}$

Dawson, M. R., Polito, A., Levine, J. M., and Reynolds, R. (2003). NG2-expressing glial progenitor cells: an abundant and widespread population of cycling cells in the adult rat CNS. Mol. Cell. Neurosci. 24, 476-488. doi: 10.1016/s10447431(03)00210-0

Dimou, L., and Simons, M. (2017). Diversity of oligodendrocytes and their progenitors. Curr. Opin. Neurobiol. 47, 73-79. doi: 10.1016/j.conb.2017.09.015

Dimou, L., Simon, C., Kirchhoff, F., Takebayashi, H., and Gotz, M. (2008). Progeny of Olig2-expressing progenitors in the gray and white matter of the adult mouse cerebral cortex. J. Neurosci. 28, 10434-10442. doi: 10.1523/JNEUROSCI.283108.2008

Djogo, T., Robins, S. C., Schneider, S., Kryzskaya, D., Liu, X., Mingay, A., et al. (2016). Adult NG2-Glia are required for median eminence-mediated leptin sensing and body weight control. Cell Metab. 23, 797-810. doi: 10.1016/j.cmet. 2016.04.013

Escartin, C., Galea, E., Lakatos, A., O’Callaghan, J. P., Petzold, G. C., SerranoPozo, A., et al. (2021). Reactive astrocyte nomenclature, definitions and future directions. Nat. Neurosci. 24, 312-325. doi: 10.1038/s41593-020-00783-4

Foerster, S., Hill, M. F. E., and Franklin, R. J. M. (2019). Diversity in the oligodendrocyte lineage: plasticity or heterogeneity? Glia 67, 1797-1805. doi: 10.1002/glia.23607

Ghorbani, S., and Yong, V. W. (2021). The extracellular matrix as modifier of neuroinflammation and remyelination in multiple sclerosis. Brain 144, 1958-1973. doi: 10.1093/brain/awab059

Givogri, M. I., Costa, R. M., Schonmann, V., Silva, A. J., Campagnoni, A. T., and Bongarzone, E. R. (2002). Central nervous system myelination in mice with deficient expression of Notch1 receptor. J. Neurosci. Res. 67, 309-320. doi: $10.1002 /$ jnr.10128

Gross, P. M., Sposito, N. M., Pettersen, S. E., and Fenstermacher, J. D. (1986). Differences in function and structure of the capillary endothelium in gray matter, white matter and a circumventricular organ of rat brain. Blood Vessels 23, 261-270. doi: 10.1159/000158652

Hagemeyer, N., Hanft, K. M., Akriditou, M. A., Unger, N., Park, E. S., Stanley, E. R., et al. (2017). Microglia contribute to normal myelinogenesis and to oligodendrocyte progenitor maintenance during adulthood. Acta Neuropathol. 134, 441-458. doi: 10.1007/s00401-017-1747-1

Hammond, T. R., Dufort, C., Dissing-Olesen, L., Giera, S., Young, A., Wysoker, A., et al. (2019). Single-cell RNA sequencing of microglia throughout the mouse lifespan and in the injured brain reveals complex cell-state changes. Immunity 50, 253-271. doi: 10.1016/j.immuni.2018.11.004

Hill, R. A., and Nishiyama, A. (2014). NG2 cells (polydendrocytes): listeners to the neural network with diverse properties. Glia 62, 1195-1210. doi: 10.1002/glia. 22664

Hill, R. A., Patel, K. D., Medved, J., Reiss, A. M., and Nishiyama, A. (2013). NG2 cells in white matter but not gray matter proliferate in response to PDGF. J. Neurosci. 33, 14558-14566. doi: 10.1523/JNEUROSCI.2001-12.2013

Itoh, N., Itoh, Y., Tassoni, A., Ren, E., Kaito, M., Ohno, A., et al. (2018). Cell-specific and region-specific transcriptomics in the multiple sclerosis model: focus on astrocytes. Proc. Natl. Acad. Sci. U S A 115, E302-E309. doi: $10.1073 /$ pnas. 1716032115

Jäkel, S., Agirre, E., Mendanha Falcao, A., van Bruggen, D., Lee, K. W., Knuesel, I., et al. (2019). Altered human oligodendrocyte heterogeneity in multiple sclerosis. Nature 566, 543-547. doi: 10.1038/s41586-019-0903-2
Junker, A., Wozniak, J., Voigt, D., Scheidt, U., Antel, J., Wegner, C., et al. (2020). Extensive subpial cortical demyelination is specific to multiple sclerosis. Brain Pathol. 30, 641-652. doi: 10.1111/bpa.12813

Kamen, Y., Pivonkova, H., Evans, K. A., and Karadottir, R. T. (2021). A matter of state: diversity in oligodendrocyte lineage cells. Neuroscientist 10:1073858420987208. doi: 10.1177/1073858420987208

Kang, S. H., Fukaya, M., Yang, J. K., Rothstein, J. D., and Bergles, D. E. (2010). NG2+ CNS glial progenitors remain committed to the oligodendrocyte lineage in postnatal life and following neurodegeneration. Neuron 68, 668-681. doi: 10.1016/j.neuron.2010.09.009

Khakh, B. S., and Sofroniew, M. V. (2015). Diversity of astrocyte functions and phenotypes in neural circuits. Nat. Neurosci. 18, 942-952. doi: 10.1038/nn.4043

Kidd, D., Barkhof, F., McConnell, R., Algra, P. R., Allen, I. V., and Revesz, T. (1999). Cortical lesions in multiple sclerosis. Brain 122, 17-26. doi: 10.1093/brain/122.1.17

Kishida, N., Maki, T., Takagi, Y., Yasuda, K., Kinoshita, H., Ayaki, T., et al. (2019). Role of perivascular oligodendrocyte precursor cells in angiogenesis after brain ischemia. J. Am. Heart Assoc. 8:e011824. doi: 10.1161/JAHA.118. 011824

Larson, V. A., Zhang, Y., and Bergles, D. E. (2016). Electrophysiological properties of NG2(+) cells: matching physiological studies with gene expression profiles. Brain Res. 1638, 138-160. doi: 10.1016/j.brainres.2015.09.010

Lassmann, H. (2019). The changing concepts in the neuropathology of acquired demyelinating central nervous system disorders. Curr. Opin. Neurol. 32, 313-319. doi: 10.1097/WCO.0000000000000685

Lassmann, H., Bruck, W., and Lucchinetti, C. (2001). Heterogeneity of multiple sclerosis pathogenesis: implications for diagnosis and therapy. Trends Mol. Med. 7, 115-121. doi: 10.1016/s1471-4914(00)01909-2

Lawson, L. J., Perry, V. H., Dri, P., and Gordon, S. (1990). Heterogeneity in the distribution and morphology of microglia in the normal adult mouse brain. Neuroscience 39, 151-170. doi: 10.1016/0306-4522(90)90229-w

Lentferink, D. H., Jongsma, J. M., Werkman, I., and Baron, W. (2018). Grey matter OPCs are less mature and less sensitive to IFNgamma than white matter OPCs: consequences for remyelination. Sci. Rep. 8:2113. doi: 10.1038/s41598018-19934-6

Li, Q., Cheng, Z., Zhou, L., Darmanis, S., Neff, N. F., Okamoto, J., et al. (2019). Developmental heterogeneity of microglia and brain myeloid cells revealed by deep single-cell RNA sequencing. Neuron 101, 207-223. doi: 10.1016/j.neuron. 2018.12.006

Ling, E. A. (1976). Some aspects of amoeboid microglia in the corpus callosum and neighbouring regions of neonatal rats. J. Anat. 121, 29-45.

Lloyd, A. F., Davies, C. L., Holloway, R. K., Labrak, Y., Ireland, G., Carradori, D., et al. (2019). Central nervous system regeneration is driven by microglia necroptosis and repopulation. Nat. Neurosci. 22, 1046-1052. doi: 10.1038/s41593-019-0418-z

Lundgaard, I., Osorio, M. J., Kress, B. T., Sanggaard, S., and Nedergaard, M. (2014). White matter astrocytes in health and disease. Neuroscience 276, 161-173. doi: 10.1016/j.neuroscience.2013.10.050

Marin-Padilla, M. (2012). The human brain intracerebral microvascular system: development and structure. Front. Neuroanat. 6:38. doi: 10.3389/fnana.2012. 00038

Marques, S., van Bruggen, D., Vanichkina, D. P., Floriddia, E. M., Munguba, H., Varemo, L., et al. (2018). Transcriptional convergence of oligodendrocyte lineage progenitors during development. Dev. Cell. 46, 504-517. doi: 10.1016/j. devcel.2018.07.005

Marques, S., Zeisel, A., Codeluppi, S., van Bruggen, D., Mendanha Falcao, A., Xiao, L., et al. (2016). Oligodendrocyte heterogeneity in the mouse juvenile and adult central nervous system. Science 352, 1326-1329. doi: 10.1126/science. aaf6463

Marzan, D. E., Brugger-Verdon, V., West, B. L., Liddelow, S., Samanta, J., and Salzer, J. L. (2021). Activated microglia drive demyelination via CSF1R signaling. Glia 69, 1583-1604. doi: 10.1002/glia.23980

Miyamoto, N., Pham, L. D., Seo, J. H., Kim, K. W., Lo, E. H., and Arai, K. (2014). Crosstalk between cerebral endothelium and oligodendrocyte. Cell. Mol. Life. Sci. 71, 1055-1066. doi: 10.1007/s00018-013-1488-9

Monje, M., and Karadottir, R. T. (2020). The bright and the dark side of myelin plasticity: neuron-glial interactions in health and disease. Semin. Cell Dev. Biol. 116, 10-15. doi: 10.1016/j.semcdb.2020.11.009 
Nishiyama, A., Shimizu, T., Sherafat, A., and Richardson, W. D. (2021). Life-long oligodendrocyte development and plasticity. Sem. Cell Dev. Biol. 116, 25-37. doi: 10.1016/j.semcdb.2021.02.004

Orduz, D., Benamer, N., Ortolani, D., Coppola, E., Vigier, L., Pierani, A., et al. (2019). Developmental cell death regulates lineage-related interneuronoligodendroglia functional clusters and oligodendrocyte homeostasis. Nat. Commun. 10:4249. doi: 10.1038/s41467-019-11904-4

Pease-Raissi, S. E., and Chan, J. R. (2021). Building a (w)rapport between neurons and oligodendroglia: reciprocal interactions underlying adaptive myelination. Neuron 109, 1258-1273. doi: 10.1016/j.neuron.2021.02.003

Pellet-Many, C., Frankel, P., Jia, H., and Zachary, I. (2008). Neuropilins: structure, function and role in disease. Biochem J. 411, 211-226. doi: 10.1042/BJ20071639

Peterson, J. W., Bo, L., Mork, S., Chang, A., and Trapp, B. D. (2001). Transected neurites, apoptotic neurons and reduced inflammation in cortical multiple sclerosis lesions. Ann. Neurol. 50, 389-400. doi: 10.1002/ana.1123

Pfeiffer, F., Sherafat, A., and Nishiyama, A. (2021). The impact of fixation on the detection of oligodendrocyte precursor cell morphology and vascular associations. Cells 10:1302. doi: 10.3390/cells10061302

Prinz, M., Masuda, T., Wheeler, M. A., and Quintana, F. J. (2021). Microglia and central nervous system-associated macrophages-from origin to disease modulation. Annu. Rev. Immunol. 39, 251-277. doi: 10.1146/annurevimmunol-093019-110159

Psachoulia, K., Jamen, F., Young, K. M., and Richardson, W. D. (2009). Cell cycle dynamics of NG2 cells in the postnatal and ageing brain. Neuron Glia Biol. 5, 57-67. doi: 10.1017/S1740925X09990354

Rivers, L. E., Young, K. M., Rizzi, M., Jamen, F., Psachoulia, K., Wade, A., et al. (2008). PDGFRA/NG2 glia generate myelinating oligodendrocytes and piriform projection neurons in adult mice. Nat. Neurosci. 11, 1392-1401. doi: 10.1038/nn.2220

Saher, G., Brugger, B., Lappe-Siefke, C., Mobius, W., Tozawa, R., Wehr, M. C., et al. (2005). High cholesterol level is essential for myelin membrane growth. Nat. Neurosci. 8, 468-475. doi: 10.1038/nn1426

Seo, J. H., Miyamoto, N., Hayakawa, K., Pham, L. D., Maki, T., Ayata, C., et al. (2013). Oligodendrocyte precursors induce early blood-brain barrier opening after white matter injury. J. Clin. Invest. 123, 782-786. doi: 10.1172/JCI65863

Sherafat, A., Pfeiffer, F., Reiss, A. M., Wood, W. M., and Nishiyama, A. (2021). Microglial neuropilin-1 promotes oligodendrocyte expansion during development and remyelination by trans-activating platelet-derived growth factor receptor. Nat. Commun. 12:2265. doi: 10.1038/s41467-021-22532-2

Spitzer, S. O., Sitnikov, S., Kamen, Y., Evans, K. A., Kronenberg-Versteeg, D., Dietmann, S., et al. (2019). Oligodendrocyte progenitor cells become regionally diverse and heterogeneous with age. Neuron 101, P459-471.E5. doi: 10.1016/j. neuron.2018.12.020

Strijbis, E. M. M., Kooi, E. J., van der Valk, P., and Geurts, J. J. G. (2017). Cortical remyelination is heterogeneous in multiple sclerosis. J. Neuropathol. Exp. Neurol. 76, 390-401. doi: 10.1093/jnen/nlx023

Sun, L. O., Mulinyawe, S. B., Collins, H. Y., Ibrahim, A., Li, Q., Simon, D. J., et al. (2018). Spatiotemporal control of CNS myelination by oligodendrocyte programmed cell death through the TFEB-PUMA axis. Cell 175, P1811-1826.E21. doi: 10.1016/j.cell.2018.10.044

Swire, M., Kotelevtsev, Y., Webb, D. J., Lyons, D. A., and Ffrench-Constant, C. (2019). Endothelin signalling mediates experience-dependent myelination in the CNS. elife 8:e49493. doi: 10.7554/eLife.49493

Tasic, B., Menon, V., Nguyen, T. N., Kim, T. K., Jarsky, T., Yao, Z., et al. (2016). Adult mouse cortical cell taxonomy revealed by single cell transcriptomics. Nat. Neurosci. 19, 335-346. doi: 10.1038/nn.4216

Terai, K., Soga, T., Takahashi, M., Kamohara, M., Ohno, K., Yatsugi, S., et al. (2003). Edg-8 receptors are preferentially expressed in oligodendrocyte lineage cells of the rat CNS. Neuroscience 116, 1053-1062. doi: 10.1016/s03064522(02)00791-1

Thion, M. S., Ginhoux, F., and Garel, S. (2018). Microglia and early brain development: An intimate journey. Science 362, 185-189. doi: 10.1126/science. aat0474

Tognatta, R., Karl, M. T., Fyffe-Maricich, S. L., Popratiloff, A., Garrison, E. D., Schenck, J. K., et al. (2020). Astrocytes Are Required for Oligodendrocyte
Survival and Maintenance of Myelin Compaction and Integrity. Front. Cell Neurosci. 14:74. doi: 10.3389/fncel.2020.00074

Tomassy, G. S., Berger, D. R., Chen, H. H., Kasthuri, N., Hayworth, K. J., Vercelli, A., et al. (2014). Distinct profiles of myelin distribution along single axons of pyramidal neurons in the neocortex. Science 344, 319-324. doi: $10.1126 /$ science. 1249766

Tsai, H. H., Niu, J., Munji, R., Davalos, D., Chang, J., Zhang, H., et al. (2016). Oligodendrocyte precursors migrate along vasculature in the developing nervous system. Science 351, 379-384. doi: 10.1126/science. aad3839

Vigano, F., Mobius, W., Gotz, M., and Dimou, L. (2013). Transplantation reveals regional differences in oligodendrocyte differentiation in the adult brain. Nat. Neurosci. 16, 1370-1372. doi: 10.1038/nn.3503

Werkman, I. L., Dubbelaar, M. L., van der Vlies, P., de Boer-Bergsma, J. J., Eggen, B. J. L., and Baron, W. (2020). Transcriptional heterogeneity between primary adult grey and white matter astrocytes underlie differences in modulation of in vitro myelination. J. Neuroinflammation 17:373. doi: 10.1186/s12974-020-02045-3

Werkman, I. L., Kovilein, J., de Jonge, J. C., and Baron, W. (2021). Impairing committed cholesterol biosynthesis in white matter astrocytes, but not grey matter astrocytes, enhances in vitro myelination. J. Neurochem. 156, 624-641. doi: 10.1111/jnc. 15113

Wlodarczyk, A., Holtman, I. R., Krueger, M., Yogev, N., Bruttger, J., Khorooshi, R., et al. (2017). A novel microglial subset plays a key role in myelinogenesis in developing brain. EMBO J. 36, 3292-3308. doi: 10.15252/embj.201 696056

Yeung, M. S. Y., Djelloul, M., Steiner, E., Bernard, S., Salehpour, M., Possnert, G., et al. (2019). Dynamics of oligodendrocyte generation in multiple sclerosis. Nature 566, 538-542. doi: 10.1038/s41586-018 $-0842-3$

Young, K. M., Psachoulia, K., Tripathi, R. B., Dunn, S. J., Cossell, L., Attwell, D., et al. (2013). Oligodendrocyte dynamics in the healthy adult CNS: evidence for myelin remodeling. Neuron 77, 873-885. doi: 10.1016/j.neuron.2013. 01.006

Zamanian, J. L., Xu, L., Foo, L. C., Nouri, N., Zhou, L., Giffard, R. G., et al. (2012). Genomic analysis of reactive astrogliosis. J. Neurosci. 32, 6391-6410. doi: 10.1523/JNEUROSCI.6221-11.2012

Zeisel, A., Munoz-Manchado, A. B., Codeluppi, S., Lonnerberg, P., La Manno, G., Jureus, A., et al. (2015). Brain structure. Cell types in the mouse cortex and hippocampus revealed by single-cell RNA-seq. Science 347, 1138-1142. doi: 10.1126/science.aaa1934

Zhu, X., Hill, R. A., Dietrich, D., Komitova, M., Suzuki, R., and Nishiyama, A. (2011). Age-dependent fate and lineage restriction of single NG2 cells. Development 138, 745-753. doi: 10.1242/dev.047951

Zilkha-Falb, R., Kaushansky, N., and Ben-Nun, A. (2020). The median eminence, a new oligodendrogenic niche in the adult mouse brain. Stem Cell Reports 14 1076-1092. doi: 10.1016/j.stemcr.2020.04.005

Conflict of Interest: The authors declare that the research was conducted in the absence of any commercial or financial relationships that could be construed as a potential conflict of interest.

Publisher's Note: All claims expressed in this article are solely those of the authors and do not necessarily represent those of their affiliated organizations, or those of the publisher, the editors and the reviewers. Any product that may be evaluated in this article, or claim that may be made by its manufacturer, is not guaranteed or endorsed by the publisher.

Copyright $\odot 2021$ Sherafat, Pfeiffer and Nishiyama. This is an open-access article distributed under the terms of the Creative Commons Attribution License (CC BY). The use, distribution or reproduction in other forums is permitted, provided the original author(s) and the copyright owner(s) are credited and that the original publication in this journal is cited, in accordance with accepted academic practice. No use, distribution or reproduction is permitted which does not comply with these terms. 